\title{
Stability of Sigma-Model Strings and Textures.
}

\author{
R S Ward* \\ Department of Mathematical Sciences, \\ University of Durham, \\ Durham DH1 3LE
}

October 27, 2018

\begin{abstract}
In flat space-time, sigma-model strings and textures are both unstable to collapse and subsequent decay. With sufficient cosmological expansion, however, they are stable in a generalized sense: a small perturbation will cause them to change their shape, but they do not decay. The current rate of expansion is sufficient to stabilize strings, but not textures.
\end{abstract}

\section{Introduction}

This letter deals with topological-soliton solutions of the $\mathrm{O}(n)$ sigma-model in curved space-time, and in particular with the effect of cosmological expansion. For topological defects with a fixed length-scale (such as abelian-Higgs strings), the effect of expansion on an individual soliton is not very significant (of course it affects the overall density of solitons, but that is not the concern here). Sigma-model solitons, however, do not have a fixed size - and so expansion can have a large effect (in particular, the conclusions of Derrick's theorem can be circumvented).

We concentrate here on three kinds of soliton, namely textures in the $\mathrm{O}(4)$ and $\mathrm{O}(3)$ systems, and strings in the $\mathrm{O}(3)$ system. So the relevant homotopy groups are $\pi_{3}\left(S^{3}\right), \pi_{3}\left(S^{2}\right)$ and $\pi_{2}\left(S^{2}\right)$ respectively. In flat space-time, any texture configuration will immediately collapse; but there exist static string solutions. The latter

*email: richard.ward@durham.ac.uk 
correspond to solutions on $\mathbf{R}^{2}$ (or more generally on a 2-surface $\Sigma$ ), which one may interpret as static straight strings extended in the $z$-direction. The (2-dimensional) energy of the configuration is bounded below by $4 \pi|N|$, where $N$ is the winding number; and one can explicitly write down the solutions which saturate this bound [1]. In fact, one can also include the back-reaction on the space-time metric, obtaining the corresponding solution of the Einstein-sigma equations; and this solution saturates a lower bound on the $C$-energy [2]. But the fact that the energy is at its topological minimum does not mean that the solution is stable. This is because the scale of the soliton is not fixed - in particular, the width of the string is not fixed. So a small perturbation may cause the string to spread out in space, or to become infinitesimally thin and decay. (Decay occurs when field gradients become large, so that the field $\vec{\phi}$ is able to climb out of its potential well where $\vec{\phi}^{2}=$ const). The only sure way to prevent the string from collapsing is to add a 'hard core' with a fixed size this is, for example, what happens for semilocal strings [3, 4, 5] where the core is an abelian-Higgs string.

In what follows below we shall see that there are exact comoving string solutions in an expanding universe, and that they are stabilized by the expansion. For textures, collapse could also be prevented by expansion, but the required rate of expansion is higher than that in the post-inflationary era. In all cases, the stability is of a generalized kind: a perturbation will cause solitons to change their shape (or size) permanently. Roughly speaking, the solitons are plastic rather than elastic.

\section{Comoving String Solutions.}

The $\mathrm{O}(3)$ sigma-model involves a unit-vector field $\vec{\phi}=\left(\phi^{1}, \phi^{2}, \phi^{3}\right)$ depending on the space-time coordinates $x^{\mu}$, with dynamics determined by the Lagrangian density $\mathcal{L}=\frac{1}{2} g^{\mu \nu}\left(\partial_{\mu} \vec{\phi}\right) \cdot\left(\partial_{\nu} \vec{\phi}\right)$. In what follows, we shall also make use of the stereographic projection $W=\left(\phi^{1}+\mathrm{i} \phi^{2}\right) /\left(1-\phi^{3}\right)$.

On a two-dimensional space $\Sigma$, the system is conformally invariant. Let us take $\Sigma$ to be either $\mathbf{R}^{2}$ (in which case we impose the boundary condition $\phi^{3} \rightarrow 1$ as $r \rightarrow \infty$ ) or $S^{2}$. If we choose local complex coordinates $(\zeta, \bar{\zeta})$ on $\Sigma$ such that its metric is $d \sigma^{2}=\Omega(\zeta, \bar{\zeta})^{2} d \zeta d \bar{\zeta}$, then any meromorphic function $W(z)$ is a solution of the field equations on $\Sigma$; and in fact all solutions on $\Sigma$ are of this form. The lower bound $E \geq 4 \pi N$ on the energy of configurations is saturated if and only if $W$ is a rational function of $\zeta$, of degree $N$.

For example, $W(\zeta)=\lambda \zeta$ denotes a 1 -soliton, located at $\zeta=0$, with arbitrary size $\lambda^{-1}>0$. So there is a potential instability: the soliton may, for example, shrink to zero size (and become singular) in finite time. In flat $(2+1)$-dimensional space- 
time, this is exactly what happens [6, 0, 8, 9]. So a straight sigma-model string in Minkowski space-time is unstable to cylindrically-symmetric perturbations: such a perturbation can cause its width to shrink to zero in finite time.

The static solution of the full coupled system was described in [2]. The energymomentum tensor is $T_{\mu \nu}=\kappa\left[\left(\vec{\phi}_{\mu} \cdot \vec{\phi}_{\nu}-\frac{1}{2} g_{\mu \nu}\left(\vec{\phi}_{\alpha}\right)^{2}\right]\right.$, the sigma-field is $W(\zeta)=\lambda \zeta$ as before, and the metric is

$$
d s^{2}=d t^{2}-d z^{2}-\frac{d \zeta d \bar{\zeta}}{\left(1+\lambda^{2}|\zeta|^{2}\right)^{16 \pi G \kappa}} .
$$

For $0 \leq 16 \pi G \kappa \leq 1$, the surface $\Sigma$ orthogonal to the string is asymptotically conical, with a fixed defect angle; the apex of the cone is smoothed out, but becomes nonsmooth in the zero-width limit $\lambda \rightarrow \infty$. Moduli-space approximations [10, 11] suggest that the string in the coupled system is unstable just as in the flat $(\kappa=0)$ case, but this is not known for certain.

These solutions can easily be generalized to an expanding universe. For example, in the $\kappa=0$ case (no back-reaction), we can take as background space-time the flat-space Robertson-Walker universe

$$
d s^{2}=d t^{2}-A(t)^{2}\left[d \zeta d \bar{\zeta}+d z^{2}\right]
$$

and then $W(\zeta)=\lambda \zeta$ is a solution representing a comoving string. In the $\kappa \neq 0$ case, $W(\zeta)=\lambda \zeta$ together with the metric

$$
d s^{2}=d t^{2}-\mathrm{e}^{2 a t}\left[d z^{2}-\frac{d \zeta d \bar{\zeta}}{\left(1+\lambda^{2}|\zeta|^{2}\right)^{16 \pi G \kappa}}\right]
$$

is a solution of the Einstein-sigma equations with cosmological constant $\Lambda=3 a^{2}$.

The moduli-space approximation referred to above consists of mechanics on the finite-dimensional moduli space $\mathcal{M}_{N}$ of static $N$-soliton solutions. In other words, we consider only a finite number of degrees of freedom, by restricting to the finitedimensional subspace $\mathcal{M}_{N}$ of the full (infinite-dimensional) configuration space of the system. For example, this was proposed for BPS monopoles [12], and in that case was shown to provide an accurate approximation [13]. It has been applied to several other systems, and in particular to the sigma-model [14, 15, 6, 16, 7, 10, 11]. In our case, the (inverse) width parameter $\lambda$ is one of the moduli, and so we may consider the dynamics of $\lambda$. The approximation is a useful one; it breaks down when radiation becomes significant, and this happens if the soliton collapses to zero width 8.

In this case, the reduced Lagrangian $L$ for dynamics on moduli space consists of a 'kinetic energy' part only — the 'potential energy' is equal to its constant topological 
value ( $4 \pi N$ above), and so is irrelevant. This kinetic energy involves an integral over $\Sigma$, which in the case $\Sigma=\mathbf{R}^{2}$ may diverge [14]. One way of dealing with this is to restrict the integral to a compact subregion of $\mathbf{R}^{2}$, such as a disc $D_{R}$ of radius $R$, where $R$ is larger than the timescale of the process being studied [7, 9]. Another way is to work on $S^{2}$, where compactness ensures convergence, and so the reduced Langrangian $L$ is well-defined. It has been has been calculated explicitly in the $N=1$ case [16]; in particular, the Langrangian for $\lambda$ is as follows. We use a parameter $\mu$

related to $\lambda$ by $\lambda=\left(\sqrt{1+\mu^{2}}+\mu\right)^{2}$. The reduced Lagrangian is $L(\dot{\mu}, \mu)=h(\mu) \dot{\mu}^{2}$, where

$$
h(\mu)=\frac{8 \pi \lambda^{2}\left[\left(\lambda^{2}+1\right) \log \lambda^{2}-2 \lambda^{2}+2\right]}{\left(1+\mu^{2}\right)\left(\lambda^{2}-1\right)^{3}} .
$$

Note that $\mu \rightarrow \infty \Leftrightarrow \lambda \rightarrow \infty$ corresponds to the soliton shrinking to zero size, while $\mu=0 \Leftrightarrow \lambda=1$ corresponds to the soliton being spread out homogeneously over $S^{2}$. In effect, $\mu$ is a dimensionless quantity which represents the ratio (size of $\left.S^{2}\right) /\left(\right.$ size of soliton); so we can model the case $\Sigma=\mathbf{R}^{2}$ (rather than $\Sigma=S^{2}$ ) by taking $\mu \gg 1 \Leftrightarrow \lambda \gg 1$.

\section{$3 \quad$ Stability of Strings.}

The aim here is to investigate the stability of sigma-model strings in a fixed background space-time. It has already been remarked that in flat space-time, the string is unstable under cylindrically-symmetric perturbations. This can easily be seen in the $\lambda$-approximation, as follows. Take $\Sigma$ to be a 2 -sphere of radius $R \gg 1$, and take $\lambda \gg 1$; so the effective Lagrangian becomes $L \approx 4 \pi R^{2} \lambda^{-4} \log (\lambda) \dot{\lambda}^{2}$. The corresponding Euler-Lagrange equation can be reduced to the quadrature

$$
\int \frac{\sqrt{\log \lambda}}{\lambda^{2}} d \lambda=K t
$$

So if $\lambda(0)=\lambda_{0}$ and $\dot{\lambda}(0)=\dot{\lambda}_{0}$ are the initial values, then the time $T$ that it takes for $\lambda(t)$ to reach $\infty$ (ie. for the string width to shrink to zero) is finite: in fact, $T \approx \lambda_{0} / \dot{\lambda}_{0}$. Comparison with numerical solution of the full system (for example, [7]) shows that even this rough approximation is a reasonable one, underestimating $T$ by around $15 \%$.

In an expanding universe, however, the string width does not shrink to zero (if the perturbation is small). For the $\lambda$-approximation, the argument is as follows. In a Robertson-Walker background, the effective Lagrangian is $L(\dot{\mu}, \mu)=A(t)^{3} h(\mu) \dot{\mu}^{2}$, and the corresponding equation of motion can be reduced to

$$
\int \sqrt{h(\mu)} d \mu=K \int A(t)^{-3} d t
$$


It follows that if $T:=\int_{t_{0}}^{\infty} A(t)^{-3} d t$ converges, and if the initial values $\mu_{0}$ and $\dot{\mu}_{0}$ satisfy

$$
A\left(t_{0}\right)^{3} \sqrt{h\left(\mu_{0}\right)} T \dot{\mu}_{0} \leq \int_{\mu_{0}}^{\infty} \sqrt{h(\mu)} d \mu
$$

(which will always be the case if $\dot{\mu}_{0}$ is small enough), then the string is stable, at least in a generalized sense. Namely, its width will not shrink indefinitely as $t \rightarrow \infty$, but will settle down to a value different from the initial one. The perturbation causes the string to shrink a bit, but then the cosmological expansion stabilizes it and it shrinks no further. So an expansion factor $A(t) \sim t^{p}$ with $p>1 / 3$ should ensure stability in this sense.

This conclusion was checked by numerical solution of the cylindrically-symmetric equation of motion, taking $\vec{\phi}$ to have the form

$$
\vec{\phi}=(\sin f \cos \theta, \sin f \sin \theta, \cos f),
$$

where $r$ and $\theta$ are polar coordinates on $\Sigma=\mathbf{R}^{2}$, and where $f=f(t, r)$. The equation for $f$ is

$$
\frac{1}{A} \frac{\partial}{\partial t}\left(A^{3} \frac{\partial f}{\partial t}\right)=\frac{1}{r} \frac{\partial}{\partial r}\left(r \frac{\partial f}{\partial r}\right)-\frac{\sin (f) \cos (f)}{r^{2}}
$$

which admits the comoving solution $f(r)=2 \cot ^{-1}(\lambda r)$. For example, taking $A(t)=$ $t^{2 / 3}$ and initial conditions (at $t=1$ ) corresponding to $\lambda_{0}=1, \dot{\lambda}_{0}=0.2$ gives a solution in which the width of the string initially shrinks, but the shrinking levels off and stabilizes. The function $f(t, r)$ as $t \rightarrow \infty$ is very close to $f(r)=2 \cot ^{-1}(\lambda r)$ with $\lambda=1.24$.

We can also consider perturbations which are localized in $z$ (rotationally- but not cylindrically-symmetric). This was done using an approximation in which the field is $W=\lambda \zeta$ with $\lambda=\lambda(t, z)$. The effective Lagrangian is

$$
L=A(t) h(\mu)\left[A(t)^{2} \mu_{t}^{2}-\mu_{z}^{2}\right]
$$

where $\mu$ and $h(\mu)$ are as defined previously. The corresponding Euler-Lagrange equations were solved numerically, with initial conditions $\mu(1, z)=10, \mu_{t}(1, z)=\operatorname{sech}(z)$. This represents making a constriction in the string, localized around $z=0$. If $A(t) \equiv 0$ (flat space-time), then the 'dent' spreads outwards from $z=0$ (at the speed of light), with $\mu(t, z)$ staying finite everywhere. With $A(t)=t^{2 / 3}$, however, the spreading-out is strongly damped: the dent remains well-localized around $z=0$, and its maximum depth (which is related to $\mu(t, 0)$ ) tends to a finite value as $t \rightarrow \infty$. Once again, we see that cosmological expansion stabilizes sigma-model strings, in the sense that a perturbation has a permanent effect, but this effect remains small. 
This discussion has assumed that the back-reaction on the space-time metric is negligible, and it leaves open the question of whether the situation changes when the back-reaction is included (or as the coupling constant $\kappa$ mentioned earlier is turned on and increased). It may be possible to extend moduli-space calculations such as those in [10, 11] so as to apply to this question.

\section{Stability of Textures.}

In this section, we investigate the stability of textures in the $O(4)$ sigma model 17 , 18, 19, 20] and the $\mathrm{O}(3)$ sigma model [21, 22, 23]. The natural tendency for textures is to shrink and eventually decay, and the question is to what extent cosmological expansion can enable them to avoid this fate. Note that $\mathrm{O}(3)$ textures are the same as closed sigma-model strings - in these loops, it is the circumference of the string which shrinks (and not its width).

Let us begin with the $\mathrm{O}(4)$ case. In an open universe, there are no comoving texture solutions. In a closed Robertson-Walker universe

$$
d s^{2}=d t^{2}-A(t)^{2}\left[d \xi^{2}+\sin ^{2} \xi\left(d \theta^{2}+\sin ^{2} \theta d \varphi^{2}\right)\right]
$$

however, a comoving solution exists [17. Let us begin by investigating $\mathrm{O}(4)$ textures in the background (4), using a $\lambda$-approximation. Take the sigma-model field $\Phi^{a}$ to have the form

$$
\Phi^{a}=(\sin f \sin \theta \cos \varphi, \sin f \sin \theta \sin \varphi, \sin f \cos \theta, \cos f),
$$

where $f=f(t, \xi)$. This represents an $\mathrm{O}(3)$-symmetric configuration. The reduced Lagrangian (after doing the $\theta$ and $\varphi$ integrations) is

$$
L=2 \pi A\left[\sin ^{2} \xi\left(A^{2} f_{t}^{2}-f_{\xi}^{2}\right)-2 \sin ^{2} f\right] .
$$

For the $\lambda$-approximation, we take $f(t, \xi)$ to be defined by $\tan (f / 2)=\lambda(t) \tan (\xi / 2)$. This may be thought of as a stereographic projection from the spatial 3-sphere to $\mathbf{R}^{3}$, followed by a dilation on $\mathbf{R}^{3}$, and then by an inverse stereographic projection to the target 3-sphere; the same configurations have been used in the study of Skyrmions [24]. Integrating over $\xi$ then leaves the effective Lagrangian

$$
L=12 \pi^{2}\left[\frac{A^{3} \dot{\lambda}^{2}}{(1+\lambda)^{4}}-\frac{A \lambda}{(1+\lambda)^{2}}\right],
$$

and the corresponding equation of motion is

$$
\ddot{\lambda}=\frac{2 \dot{\lambda}^{2}}{1+\lambda}-\frac{3 \dot{A} \dot{\lambda}}{A}-\frac{1-\lambda^{2}}{2 A^{2}}
$$


which is the same as

$$
\frac{d}{d t}\left[\frac{A^{3} \dot{\lambda}}{(1+\lambda)^{3}}\right]=\frac{A(\lambda-1)}{2(\lambda+1)} .
$$

The comoving solution referred to earlier is $\lambda(t) \equiv 1$, representing a spatiallyhomogeneous texture. If $A$ is constant (no expansion), then this solution is unstable, as is clear from (7) (this contradicts the claim for stability made in [17]). Note that the system is invariant under $\lambda \mapsto \lambda^{-1}$; that collapse corresponds to either $\lambda \rightarrow 0$ or $\lambda \rightarrow \infty$; and that $\lambda$ ( or $\lambda^{-1}$ ) may be thought of as the ratio between the size of physical space and the size of the texture. So in particular for $\lambda$ close to zero, the spatial curvature should play little role, and the results should provide an indication of what happens in an open universe.

Let us now investigate the behaviour of solutions of (8) in an expanding universe. If the texture is highly localized (say $0<\lambda \ll 1$ ), then (8) can be approximately solved, as follows. Taking $A(t) \sim t^{p}$, we get $\lambda(t) \approx \alpha+\beta t^{1-3 p}+\gamma t^{2-2 p}$, where $\alpha$, $\beta$ and $\gamma$ are constants. It follows from this that if $p \leq 1$, then $\lambda$ will inevitably become zero (ie. the texture will collapse and decay) in finite time; but if $p>1$, then (depending on initial conditions) it might survive indefinitely. In particular, in our current universe, small textures certainly collapse.

What about the homogeneous texture, with $\lambda=1$ ? Numerical solution of (7) shows that the same is true. Namely, start with $\lambda=1$ and put in a (small) perturbation which acts to reduce $\lambda$. Then for $p \leq 1, \lambda(t)$ reaches 0 in finite time; while for $p>1, \lambda(t) \rightarrow \lambda_{\infty}$ as $t \rightarrow \infty$, where $0<\lambda_{\infty}<1$.

We can confirm this picture by studying $\mathrm{O}(3)$-symmetric textures in a flat-space Robertson-Walker background. The relevant reduced Lagrangian in this case is

$$
L=2 \pi A\left[r^{2}\left(A^{2} f_{t}^{2}-f_{r}^{2}\right)-2 \sin ^{2} f\right],
$$

where $f=f(t, r)$ with boundary conditions $f(t, 0)=\pi$ and $f(t, \infty)=0$. If $A(t) \equiv 1$, the equations of motion admit the well-known 'collapsing' solution $f=2 \cot ^{-1}(-r / t)$ for $t<0$ [19]. In the expanding case, the equations can be solved numerically, and the result is as before: if $A(t) \sim t^{p}$ with $p>1$, then a small perturbation causes textures to change their shape, but they do not collapse.

For Hopf textures, ie. for the $\mathrm{O}(3)$ sigma model, one expects the results to be very similar. Imposing an ansatz analogous to (5) is no longer very natural, since Hopf structures cannot be $\mathrm{O}(3)$-symmetric ( $c f$. [23]). But there is a natural oneparameter family of configurations labelled by $\lambda$ (cf. [25]), leading to an equation similar to (8), and to the same conclusions. The configuration with $\lambda=1$ is an exact comoving spatially-homogeneous solution (in closed Robertson-Walker space-time); 
it consists simply of the standard Hopf map from the spatial $S^{3}$ to the target $S^{2}$. So this static solution is unstable, and all other Hopf textures inevitably collapse, if the cosmological expansion is slower $A(t) \sim t$; but during an era of faster expansion, they are stable in a generalized sense.

\section{References}

[1] A Belavin and A Polyakov, Metastable states of two-dimensional isotropic ferromagnets. JETP Lett 22 (1975) 245-247.

[2] A Comtet and G W Gibbons, Bogomol'nyi bounds for cosmic strings. Nucl Phys B 299 (1988) 719-733.

[3] T Vachaspati and A Achúcarro, Semilocal cosmic strings. Phys Rev D 44 (1991) 3067-3071.

[4] M Hindmarsh, Existence and stability of cosmic strings. Phys Rev Lett 68 (1992) $1263-1266$.

[5] R A Leese, The stability of semilocal vortices at critical coupling. Phys Rev D 46 (1992) 4677-4684.

[6] R A Leese, M Peyrard and W J Zakrzewski, Soliton stability in the $\mathrm{O}(3)$ sigma model in (2+1) dimensions. Nonlinearity 3 (1990) 387-412.

[7] B Piette and W J Zakrzewski, Shrinking of solitons in the (2+1)-dimensional $S^{2}$ sigma model. Nonlinearity 9 (1996) 897-910.

[8] P Bizon, T Chmaj and Z Tabor, Formation of singularities for equivariant (2+1)dimensional wave maps into the 2-sphere. Nonlinearity 14 (2001) 1041-1053.

[9] J M Linhart and L A Sadun, Fast and slow blowup in the $S^{2}$ sigma model and (4+1)-dimensional Yang-Mills model. math-ph/0105024

[10] J M Speight and I A B Strachan, Gravity thaws the frozen moduli of the $C P^{1}$ lump. Phys Lett B 457 (1999) 17-22.

[11] J Gutowski, The moduli space of $C P^{1}$ stringy cosmic lumps. hep-th/0109123

[12] N S Manton, A remark on the scattering of BPS monopoles. Phys Lett B 110 (1982) 54-56.

[13] D Stuart 1994 The geodesic approximation for the Yang-Mills-Higgs equations. Commun Math Phys 166 149-190.

[14] R S Ward, Slowly-moving lumps in the $\mathrm{CP}^{1}$ model in $(2+1)$ dimensions. Phys Lett B 158 (1985) 424-428. 
[15] R A Leese, Low-energy scattering of solitons in the $\mathrm{CP}^{1}$ model. Nucl Phys B 344 (1990) 33-72.

[16] J M Speight, Low-energy dynamics of a $\mathrm{CP}^{1}$ lump on the sphere. J Math Phys 36 (1995) 796-813.

[17] R L Davis, Texture: a cosmological topological defect. Phys Rev D 35 (1987) 3705-3708.

[18] N Turok, Global texture as the origin of cosmic structure. Phys Rev Lett 63 (1989) 2625-2628.

[19] N Turok and D N Spergel, Global texture and the microwave background. Phys Rev Lett 64 (1990) 2736-2739.

[20] D N Spergel, N Turok, W H Press and B S Ryden, Global texture as the origin of large-scale structure: numerical simulations of evolution. Phys Rev D 43 (1991) 1038-1046.

[21] X Luo, Toroidal symmetric global texture in $\mathrm{O}(3)$ sigma models. Phys Lett B 287 (1992) 319-324.

[22] A Sornborger, S M Carroll and T Pyne, Collapse of exotic textures. Phys Rev D 55 (1997) 6454-6465.

[23] E W Hirschmann and S L Liebling, Pair production in the collapse of a Hopf texture. Class Quant Grav 16 (1999) 823-840.

[24] N S Manton, Geometry of Skyrmions. Commun Math Phys 111 (1987) 469-478.

[25] R S Ward, Hopf solitons on $S^{3}$ and $\mathbf{R}^{3}$. Nonlinearity 12 (1999) 241-246. 